13. Van Dyke T, Van Winkelhoff A. Infection and inflammatory mechanisms. J Clin Periodontol. 2013; 40(14): 1-7.

14. Preshaw P, Alba A, Herrera D, Jepsen S, Konstantinidis A, Makrilakis K. Periodontitis and diabetes: a two-way relationship. Diabetología. 2012; 55 (1): 21-3.

15. Colchado Jorge R. Alteraciones en la fagocitosis de los leucocitos creviculares de pacientes diabéticos con enfermedad periodontal. Odontol Sanmarquina. 2008;11(1):14-8.

16. Bullon P, Morillo J, Ramirez-Tortosa M. Metabolic syndrome and periodontitis: is oxidative stress a common link? J Dent Res. 2009; 88:503-518.

17. Thanakun $\mathrm{S}$, Watanabe $\mathrm{H}$, Thaweboon $\mathrm{S}$, Izumi Y. Association of untreated metabolic syndrome with moderate to severe periodontitis in Thai population. J Periodontol. 2014; 85:1502-1514.

18. Zuk A, Quiñonez C, Lebenbaum M. The association between undiagnosed glycemic abnormalities and cardiometabolic risk factors with periodontitis: results from 2007-2009 canadian health measures survey. J Clin Periodontol. 2017; 44:132-141

19. D'Aiuto F, Sabbah W, Netuveli G. Association of the metabolic syndrome with severe periodontitis in a large U.S. populationbased survey. J Clin Endocrinol Metab. 2008; 93:3989-3994.

20. Nesbitt M, Reynolds MA, Shiau H, Choe K, Simonsick EM, Ferrucci L. Association of periodontitis and metabolic syndrome in the Baltimore Longitudinal Study of Aging. Aging Clin Exp Res. 2010; 22:238-242.

21. Han D, Shin H, Kim M. Group of serum inflammatory markers and periodontitis-metabolic syndrome coexistence in Koreans. J Periodontol. 2012; 83:612-620.

22. Kaye E, Chen N, Cabral H, Vokonas P, Garcia R. Metabolic Syndrome and Periodontal Disease Progression in Men. J Dent Res. 2016; 95(7): 822-828.

23. Alhabashneh $R$, Khader $Y$, Herra $Z$, et al. The association between periodontal disease and metabolic syndrome among outpatients with diabetes in Jordan. J Diabetes Metab Disord. 2015; 14:67

24. Li Y, Lu Z, Zhang X. Metabolic syndrome exacerbates inflammation and bone loss in periodontitis. J Dent Res. 2015; 94:362-370

25. Genco R, Grossi S, Ho A, Nishimura F, Murayama Y. A proposed model linking inflammation to obesity, diabetes, and periodontal infections. J Periodontol. 2005; 76(11): 2075-2084.

26. Fenesy K. Periodontal diseases: An overview for physicians. Mt Sinai J Med. 1998; 65:362-9.

27. Mohammadi M, Gozashti MH, Aghadavood M, Mehdizadeh MR, Hayatbakhsh MM. Clinical Significance of Serum IL-6 and TNF- $\alpha$ Levels in Patients with Metabolic Syndrome. Rep Biochem Mol Biol. 2017;6(1):74-79.

28. Gay I, Tran D, Cavender A, Weltman R, Chang J, Luckenbach E, et al. The effect of periodontal therapy on glycemic control in a hispanic population with type 2 diabetes: $A$ randomized controlled trial. J Clin Periodontol. 2014; 41:673-80. 
29. Gallagher $E$, Leroith D, Karnieli E. The metabolic syndrome-from insulin resistance to obesity and diabetes. Med Clin North Am. 2011; 95(5):855- 873 


\title{
Ansiedad al tratamiento odontológico en adultos mayores a 65 años, de la parroquia Cañaribamba, Cuenca-Ecuador, 2017-2018
}

\section{Dental treatment anxiety in adults over 65 years of age, from Cañaribamba parish, Cuenca-Ecuador, 2017-2018.}

\author{
Alejandra Naranjo", Santiago Vintimilla², Patricia Pinos². \\ 1 Estudiante de Odontología en la Universidad Católica de Cuenca. \\ 2 Docente de Odontología en la Universidad Católica de Cuenca. \\ *mayale93@hotmail.es
}

DOI: https://

\section{Resumen}

Objetivo: Determinar la prevalencia de ansiedad al tratamiento odontológico en adultos mayores a 65 años, de la parroquia Cañaribamba, Cuenca-Ecuador, agosto 2017- abril 2018. Materiales y Métodos: Se realizó un estudio de enfoque cuantitativo y descriptivo, de campo, comunicacional y retrospectivo. Se evaluó a 320 personas mayores a 65 años (114 mujeres y 206 hombres), las mismas que llenaron el test de auto reporte escala de ansiedad dental versión corta. Las encuestas se realizaron de manera aleatoria a los habitantes de la parroquia de Cañaribamba. Resultados: Se determinó una prevalencia de ansiedad al tratamiento odontológico con un 58\%. El nivel de ansiedad leve fue el que mayor predominio tuvo en la población $(27,19 \%)$, seguido por la ansiedad moderada $(15,31 \%)$. El nivel de ansiedad predominante en la mujeres fue el leve con un $35,96 \%$, al igual que en los hombres con el $22,33 \%$. Existe una relación estadísticamente significativa $(a \quad p<0,05)$ entre el género y el nivel de ansiedad dental $\left(X^{2} p=0,0068\right)$. Las principales causas que provocaron ansiedad en los adultos mayores fueron en el momento en que el odontólogo alista el carpule con anestesia $(19,28 \%)$, pensar que 
el odontólogo va a utilizar la turbina en su boca $(16,05 \%$ ), y pensar que el odontólogo le va a extraer el diente (15,70\%). Conclusión: Se concluyó que existe un alto porcentaje de ansiedad al tratamiento odontológico en los adultos mayores a 65 años (58\%), respecto a estudios realizados en países más desarrollados ( $4 \%$ a $36 \%$ ).

Palabras clave: Prevalencia, ansiedad, causas, tratamiento.

\section{Abstract}

Objective: To determine the prevalence of anxiety in dental treatment in adults over 65 years of age, from the Cañaribamba Parish, Cuenca - Ecuador, August 2017-April 2018. Materials and Methods: Quantitative and descriptive approach, field study, communicational and retrospective. A total of 320 people over 65 years of age were evaluated (114 women and 206 men), who completed the self-report SDAI (dental anxiety scale short version). The surveys were carried out in a random way to the inhabitants of the parish of Cañaribamba. Results: A prevalence of anxiety in dental treatment was determined with $58 \%$. The level of anxiety was higher in the population $(27,19 \%)$, followed by moderate anxiety $(15,31 \%)$. The predominant level of anxiety in women was $35,93 \%$, as in men with $22,33 \%$. There is a statistically significant relationship $(\alpha<0.05)$ between the gender and the level of dental anxiety $(X 2=0,0068)$. The main factors that caused anxiety in the elderly were at the time when the dentist enrolled the carpule with anesthesia $(19,28 \%)$, see that the dentist will use the turbine in his mouth $(16,05 \%)$, and think that the dentist is going to extract the tooth $(15,70 \%)$. Conclusion: It concluded that there is a high percentage of anxiety in dental treatment in adults over 65 years (58\%), compared to studies conducted in more up to date countries ( $4 \%$ to $36 \%$ ).

Keywords: Prevalence, anxiety, causes, treatment. 


\section{Introducción}

En la actualidad las enfermedades bucodentales son un problema de salud pública, debido a su elevada prevalencia y el costo que implica recurrir a una atención odontológica. ${ }^{1-3}$ Los fac- $^{-}$ tores que influyen en los pacientes durante el tratamiento odontológico es la preocupación de sentir dolor, ser herido, atorarse 0 ahogarse, incluso las experiencias traumáticas que pudo haber tenido en la niñez, inducen a los pacientes a postergar sus citas interrumpiendo el tratamiento, o por último a la anulación. ${ }^{8-10}$ Estos comportamientos negativos son las señales de un paciente temeroso ${ }^{8}$, es importante considerar a tiempo el nivel de ansiedad que adquirió el paciente de manera que si no se trata este fenómeno a tiempo, corren el riesgo de tener problemas de salud oral, ${ }^{8-10,17,19}$ como la caries y la enfermedad periodontal, teniendo como consecuencias físicas, económicas, sociales y psicológicas, interfiriendo en la calidad de vida de los pacientes debido a la inasistencia a sus citas, y su retraso a la eficacia del tratamien-

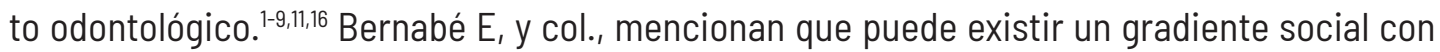
aquellos de grupos socioeconómicos más bajos que experimentan más ansiedad dental y por lo tanto más caries dental. ${ }^{1-}$

Luego de identificar a un paciente ansioso o temeroso, puede ponerse en funcionamiento una serie de medidas, para que pueda ser atendido en el área odontológica, el odontólogo deberá empezar por tratamientos simples como una profilaxis o limpieza dental, utilizando técnicas de relajación, respiración, y distracción, de manera que el miedo comience a desaparecer., 816,23

Existen pocos estudios de la situación actual de salud oral en nuestra sociedad, motivo por el cual fue necesario hacer un estudio descriptivo epidemiológico, con el propósito de saber la magnitud del problema de ansiedad frente al tratamiento odontológico en la parroquia Cañaribamba de la ciudad de Cuenca.

Con la elaboración de este estudio se determinó cuál es la prevalencia de ansiedad frente al tratamiento odontológico en adultos mayores a 65 años. Se utilizó la escala de ansiedad dental versión corta (SDAI de Stouthard), una escala de 9 ítems con respuestas dicotómicas (SI-NO), que clasifica la ansiedad de los participantes ante la perspectiva de una visita al dentista, cuando los pacientes están en la sala de espera, pensar que el odontólogo va a utilizar la turbina en su boca, en el momento en que el odontólogo alista el carpule con anestesia. Las respuestas variaron de no ansiosas (0) a extremadamente ansiosas ( $>0)$.

El presente tema de investigación surgió a partir de la continua búsqueda de información de la línea basal planteado por el departamento de investigación de la carrera de Odontología de la Universidad Católica de Cuenca, para poder establecer técnicas que nos ayuden a disminuir la ansiedad de los pacientes en nuestro medio, promoviendo atención primaria en salud oral, y de esta manera evitar complicaciones futuras de salud oral. 


\section{Materiales y métodos}

Es un estudio de enfoque cuantitativo y descriptivo, de campo, comunicacional, participativo y retrospectivo. Que permitió obtener importante información sobre aspectos relacionados con la ansiedad frente al tratamiento odontológico. ${ }^{31}$

La investigación se realizó entre los meses agosto 2017- abril 2018, recolectando datos de encuestas que reflejaron el nivel de ansiedad y las causas más relevantes frente al tratamiento odontológico en la población de adultos mayores de 65 años, que tengan la voluntad de autorizar su entrevista previa al consentimiento informado.

Una vez terminadas las encuestas los datos fueron ingresados a una base de datos llamado programa EPI INFO, los mismos que fueron utilizados para analizarlos a través del programa Excel Microsoft Office.

La población de estudio fue de "320" fichas epidemiológicas realizadas a (114 mujeres y 206 hombres) de la parroquia Cañaribamba, que corresponde al total de fichas registradas en el estudio epidemiológico de salud bucal para esta parroquia.

La investigación de ansiedad al tratamiento odontológico busco describir cualitativamente el problema en adultos mayores de 65 años de edad, utilizando las fichas obtenidas por encuestas realizadas por estudiantes de la Universidad Católica de Cuenca en el periodo agosto 2017- abril 2018.

Los Criterios de evaluación de ansiedad se basaron en la puntuación de respuesta.

Las condiciones que se tomaron en cuenta a la hora del registro de los datos recolectados fueron:

- Puede indicar una respuesta por ítem.

- Se sumarán los puntajes de la escala de ansiedad al tratamiento odontológico.

- El rango de puntaje irá de 0 a 1 .

- Donde $>0$ es igual a ansiedad y 0 es igual a sin ansiedad.

El puntaje mayor estará asociado con niveles altos de ansiedad al tratamiento odontológico.

Para tener el valor de ansiedad se tomó en cuenta en las preguntas donde (SI) es igual a (1), Y (NO) es igual a (0).

Se realizó un análisis descriptivo de las variables, en la primera variable se determinará la prevalencia de ansiedad, en cuanto al segunda variable que es la población del estudio, se analizara con Chi cuadrado $X^{2}$ la asociación entre género y el nivel de ansiedad. 


\section{Resultados}

El presente estudio fue realizado con los datos epidemiológicos de fichas de recolección de información digital que constan en el archivo del departamento de investigación de la Unidad de Salud y Bienestar Carrera de Odontología de la Universidad Católica de Cuenca. Datos de encuestas que reflejaron el nivel de ansiedad y las causas más relevantes frente al tratamiento odontológico en población de adultos mayores a 65 años, mostrando los siguientes resultados:

\begin{tabular}{lll}
\hline Ansiedad & $\mathbf{n}$ & $\%$ \\
\hline SI & 185 & 58 \\
No & 135 & 42 \\
Total general & 320 & 100 \\
\hline
\end{tabular}

Tabla №1. Distribución de la muestra de acuerdo a la prevalencia de ansiedad frente al tratamiento odontológico en adultos mayores a 65 años de la parroquia Cañaribamba agosto 2017- abril 2018.

Se evidencia la prevalencia del tamaño de muestra. Observamos que de los 320 adultos mayores a 65 años encuestados, 185 presentan ansiedad y 135 de ellos sin ansiedad.

\begin{tabular}{lll}
\hline $\begin{array}{l}\text { Niveles de } \\
\text { Ansiedad }\end{array}$ & $\mathbf{n}$ & $\%$ \\
\hline Severa & 49 & 15.31 \\
Moderna & 49 & 15.31 \\
Leve & 87 & 27.19 \\
No tiene ansiedad & 135 & 42.19 \\
Total general & $\mathbf{3 2 0}$ & $\mathbf{1 0 0}$ \\
\hline
\end{tabular}

Tabla № 2. Niveles de ansiedad en adultos mayores a 65 años frente al tratamiento odontológico.

En este gráfico se puede observar que existe ansiedad ante el tratamiento odontológico, predominando un nivel de ansiedad leve con $27 \%$, seguida del nivel moderada con $15 \%$ y un $15 \%$ presenta ansiedad severa al dentista o tratamientos dentales, y gran parte de los pacientes no lo padecen,representando un $42 \%$ sin ansiedad de la parroquia Cañaribamba.

\begin{tabular}{cccccc}
\hline Niveles de Ansiedad & \multicolumn{2}{c}{ Femenino } & \multicolumn{2}{c}{ Masculino } & Total general \\
\hline Severa & 22 & 19,30 & 27 & 13,11 & 49 \\
Moderna & 12 & 10,53 & 37 & 17.96 & 49 \\
Leve & 41 & 35,96 & 46 & 22,33 & 87 \\
No tiene ansiedad & 39 & 34,21 & 96 & 46,60 & 135 \\
Total general & $\mathbf{1 1 4}$ & $\mathbf{1 0 0 . 0 0}$ & $\mathbf{2 0 6}$ & $\mathbf{1 0 0 . 0 0}$ & $\mathbf{3 2 0}$ \\
\hline
\end{tabular}

Tabla № 3. Distribución de la muestra según el género y el nivel de ansiedad dental durante la atención odontológica. 
Se puede evidenciar que predomina el género masculino con un $46 \%$ que no experimenta ansiedad, en relación con el género femenino con un $34 \%$ no tiene ansiedad durante la atención odontológica.

Existe un mayor indice de personas del género femenino con un nivel de ansiedad leve. El Chi 2 indico que hay relación estadísticamente significativa entre el género y el nivel de ansiedad dental.

\begin{tabular}{|c|c|c|}
\hline № & CAUSAS & $\%$ \\
\hline 1 & $\begin{array}{l}\text { En el momento en que el } \\
\text { odontólogo alista la jeringa con } \\
\text { inyección de anestesia Pensar } \\
\text { que el odontólogo va }\end{array}$ & 19.28 \\
\hline 2 & $\begin{array}{l}\text { a utilizar el taladro en su } \\
\text { boca }\end{array}$ & 16.05 \\
\hline 3 & $\begin{array}{l}\text { Pensar que el odontólogo le } \\
\text { va a extraer el diente }\end{array}$ & 15.70 \\
\hline 4 & $\begin{array}{l}\text { No conocer lo que el } \\
\text { odontólogo se encuentra } \\
\text { realizando en su boca } \\
\text { Cuando el odontólogo le }\end{array}$ & 11.37 \\
\hline 5 & $\begin{array}{l}\text { invita a sentarse en la silla } \\
\text { dental }\end{array}$ & 8.90 \\
\hline 6 & $\begin{array}{l}\text { Dejar el consultorio al penser } \\
\text { que el odontólogo no le va a } \\
\text { explicar el problema que } \\
\text { tiene en sus dientes }\end{array}$ & 8.28 \\
\hline 7 & $\begin{array}{l}\text { Suda y tiembla cuando es su } \\
\text { turno de pasar a la consulta } \\
\text { Pensar que va a estar } \\
\text { sentado en la silla dental }\end{array}$ & 6.92 \\
\hline 8 & $\begin{array}{l}\text { para un tratamiento le pone } \\
\text { nervioso }\end{array}$ & 6.80 \\
\hline 9 & $\begin{array}{l}\text { Mientras camins al } \\
\text { consultorio piensa que el } \\
\text { odontólogo no le va a } \\
\text { explicar el problema } \\
\text { que tiene en sus dientes }\end{array}$ & 6.67 \\
\hline
\end{tabular}

Tabla № 4. Causas más relevantes que influyen en la ansiedad frente al tratamiento odontológico. 


\section{Discusión}

En este estudio se pudo evidenciar que existe un mayor porcentaje de la población que presenta ansiedad al tratamiento odontológico con un $58 \%$, a diferencia de la investigación de Ríos Erazo M, et al, realizado en Santiago de Chile, indicaron bajas prevalencias de ansiedad dental en adultos chilenos que oscilaban entre los $20-70$ años, presentando un $37,9 \%,{ }^{7}$ siendo porcentajes menores en comparación de este estudio que presenta un $20 \%$ mayor de ansiedad. En el estudio de Ríos Erazo no se realizó la distribución de los niveles de ansiedad por sexo, ${ }^{7}$ a diferencia de esta investigación en el que si se distribuye la muestra de acuerdo al nivel de ansiedad según el género.

El porcentaje de ansiedad según el género que presenta este estudio es de 35,9\% mayor para el género femenino. Este estudio tiene relación con la investigación realizada por Martínez, et al, en que indican un porcentaje de ansiedad de $48,2 \%$ mayor para género femenino. ${ }^{10}$ Relacionando estos dos estudios, se puede evidenciar que existe una concordancia de ansiedad según el género, predominando el género femenino con mayor ansiedad. En el estudio de Martínez, se realizó una encuesta mediante un test de ansiedad dental de Norman Corah, ${ }^{10} \mathrm{a}$ diferencia de este estudio que se utilizó la escala de ansiedad dental versión corta SDAl, escala que nos permite saber mediante un test la ansiedad dental que experimentan los pacientes durante la consulta odontológica.

Al comparar este estudio con una investigación de Ríos Erazo M, et al. Indican que el nivel de ansiedad más frecuente observado es el moderado con un $16,1 \%$. Sus resultados son similares a este estudio el cual presentó un 15,31\% nivel de ansiedad moderado. ${ }^{7}$ A diferencia de otro estudio realizado por Martínez López, donde indica porcentajes más altos en comparación a otros estudios, presentando un $28,8 \%$ de ansiedad moderada. ${ }^{10}$

Es importante que el odontólogo considere a tiempo el nivel de ansiedad que adquirió el paciente, de manera que si no se trata este fenómeno a tiempo, corren el riesgo de tener problemas de salud oral interfiriendo en la calidad de vida de los pacientes debido a la inasistencia a sus citas, y su retraso a la eficacia del tratamiento odontológico.

El odontólogo debe aplicar una serie de medidas básicas, usando técnicas de relajación, respiración, y distracción, de manera que el miedo comience a desaparecer y que los pacientes ansiosos puedan ser atendidos en el área odontológica.

\section{Conclusiones}

Al concluir el estudio podemos decir que la prevalencia de ansiedad en adultos mayores a 65 años de la parroquia de Cañaribamba, Cuenca-Ecuador, se encuentra con un porcentaje alto (58\%) respecto a estudios realizados en países más desarrollados ( $4 \%$ a $36 \%$ ).

La ansiedad leve es uno de los niveles más altos que presentan los adultos mayores, seguida por la ansiedad moderada, siendo estos porcentajes altos para este estudio pero bajos para 
otros estudios, y por último se encuentra la ansiedad severa con un porcentaje mayor a diferencia de otros estudios.

El género femenino presenta porcentajes más altos según el nivel de ansiedad leve, moderada y severa, a diferencia del género masculino que presentan valores bajos.

Las causas de mayor predominio de ansiedad reflejadas en este estudio fueron, el momento en que el odontólogo alista la jeringa con inyección de anestesia, pensar que se va a utilizar la turbina en su boca y cuando el profesional le va a extraer el diente. Causas por la que la mayoría de pacientes referían miedo o temor al momento de la consulta 0 en tratamientos odontológicos. Estas preguntas fueron realizadas en el test de la escala de ansiedad dental versión corta SDAl.

\section{Referencias Bibliográficas:}

1. Bernabé $E$, Humphris $G$, Freeman R. The social gradient in oral health: Is there a role for dental anxiety. Community Dent Oral Epidemiol. 2017 Aug;45(4):348-355. doi: 10.1111/ cdoe.12297. Epub 2017 Mar 28. Disponible en: https://onlinelibrary.wiley.com/doi/ pdf/10.1111/cdoe.12297

2. OMS. Nota informativa $\mathrm{N}^{\circ} 318$. I Salud bucodental. Who. 2012. p. 1. Disponible en: http:// www.who.int/mediacentre/factsheets/fs318/es/.

3. Ingunza, J., et al. Calidad de vida relacionada a la salud bucal en escolares de ámbito urbano-marginal. Rev Estomatol Herediana. 2015 Jul-Set;25(3):194-204.

4. Real Academia Española de la Lengua. Ansiedad [Internet]. Diccionario de la lengua española. 2014. Disponible en: https://www.mendeley.com/research-papers/ calidad-vida-relacionada-la-salud-bucal-en-escolares-\%C3\%A1mbito-urbanomarginal-tt-oral-health-relatedqu/?utm_source=desktop\&utm_medium $=1.17 .13 \& u t m \_c a m-$ paign=open_catalog\&userDocumentld=\%7B490a6c2b-ccf0-46fe-b69a-34daed0524bc\%7D.

5. Pozo Bassi J, Pavez Tetlak C, Riquelme Tapia D, Quiroga del Pozo J. Comparación en los niveles de ansiedad en pacientes previo a la realización de terapia endodóntica y periodontal. Rev Clínica Periodoncia, Implantol y Rehabil Oral [Internet]. 2015;8(3):20812. Disponible en: https://scielo.conicyt.cl/scielo.php?script=sci_arttext\&pid=S 0718381X2016000200012

6. Amaíz Flores AJ, Flores MÁ. Abordaje de la ansiedad del paciente adulto en la consulta odontológica: propuesta interdisciplinaria. Odontol Vital [Internet]. 2016;1(24):21-8. Disponible en: http://www.scielo.sa.cr/pdf/odov/n24/1659-0775-odov-24-00021.pdf

7. Ríos-Erazo M, Herrera-Ronda A, Molina-Muñoz Y, Et al. Ansiedad dental en adultos chilenos que concurren a un servicio de salud primaria. Int. J. Odontostomat. [Internet]. 2016 Ago [citado 2018 Jun 27]; 10 (2): 261-266. Disponible en: https://scielo.conicyt. $\mathrm{cl} /$ scielo.php?script=sci_arttext\&pid=S0718-381X2016000200012\&lng=es. http://dx.doi. org/10.4067/S0718381X2016000200012. 
8. Hmud R, Lj W. Dental anxiety: causes, complications and management approaches. J Minim Interv Dent. 2009;2(1):67-78. Disponible en:https://www.researchgate.net/publication/237649777_Ansiedad_dental_causas_complicaciones_y_metodos_de_manejo

9. Caycedo C, Cortés OF, Gama R, Rodríguez H, Colorado P, Caycedo M, et al. Ansiedad al tratamiento odontológico: características y diferencias de género. Suma Psicológica Bogotá Suma Psicológica. 2008;15(15):259-78. Disponible en:http://www.redalyc.org/ articulo.oa?id=134212604011

10. Martínez López C, Et al. niveles de ansiedad en la consulta odontológica en pacientes adultos atendidos en la universidad santo tomás. Rev UstaSalud. D 13 (2), pp. 112-119, julio-diciembre 2014. Bucaramanga. Disponible en: http://revistas.ustabuca.edu.co/index.php/USTASALUD_ODONTOLOGIA/article/viewFile/1729/1319

11. Arrieta Vergara K, Díaz Cárdenas S, Verbel Bohórquez J, Hawasly Pastrana N. Factores asociados a sintomatología clínica de miedo y ansiedad en pacientes atendidos en Odontología. Rev clín med fam. 2013;6(1):17-24. Disponible en: http://scielo.isciii.es/ scielo.php?script=sci_arttext\&pid=S1699-695X2013000100004

12. Benítez-Salazar M, Montoya-Cañón S. Frecuencia de asistencia a la consulta odontológica en estudiantes de secundaria de Pereira y los factores emocionales asociado. Rev Nac Odontol. 2015;11(21):75-81. Disponible en: http:// dx.doi.org/10.16925/od.v11i21.938

13. Diaz $L$, Romina $A$, Nivel de ansiedad en pacientes de 18 a 65 años de edad atendido en la Clinica Estomatologica de la Uiversidad Privada Antenor Orrego - Trujillo, Noviembre 2015, Universidad Privada Antenor Orrego - UPAO, 28-oct 2916. Disponible en: http:// repositorio.upao.edu.pe/handle/upaorep/2024

14. Álvarez V, Lourdes P, Narváez L, Geovanna G, Evaluación del nivel de ansiedad en pacientes pediátricos que acuden a la Facultad de Odontología de la Universidad Central del Ecuador mediante estimulación musical, 2015, Editorial : Quito: UCE, Disponible en: http://www.dspace.uce.edu.ec/handle/25000/4083

15. Bermúdez P, Támara A, William J, Eficacia y seguridad de clonidina versus placebo para ansiedad en odontología, Revista Nacional de Odontologia, , Ediciones Universidad Cooperativa de Colombia, Vol. 9, Núm. 17 (2013), Disponible en: https://doi.org/10.16925/od. v9i17.550

16. Pozo J, Pavez C, Riquelme T. Quiroga J, Comparación en los niveles de ansiedad en pacientes previo a la realización de terapia endodóntica y periodontal, Revista Clínica de Periodoncia, Implantología y Rehabilitación Oral, Volume 8, Issue 3, December 2015, Pages 208-212, Disponible en: http://www.sciencedirect.com/science/article/pii/ S0718539115000701

17. Ordóñez M, Villavicencio $E$, Alvarado 0 , Vanegas $M$, Prevalencia de bruxismo de vigilia evaluado por auto-reporte en relación con estrés, ansiedad y depresión, Revista Estomatológica Herediana, Rev. Estomatol. Herediana vol.26 no.3 Lima jul. 2016, Disponible en: http://www.scielo.org.pe/scielo.php?pid=\$101943552016000300005\&script=sci_ arttext\&tlng=pt

18. Manrique $C$, César $E$, Niveles de ansiedad en sujetos que practican el malabarismo en la ciudad de Lima Metropolitana, Repositorio Institucional, 2017-08-04, Disponible en: http://repositorio.uigv.edu.pe/handle/20.500.11818/1396 
19. Díaz E, Dimensiones del miedo dental, Revista Europea de Odontológica, Publicado el: 11/01/2017 09:29:48, Disponible en: http://www.redoe.com/ver.php?id=243

20. Chaves I, Dr. Silva B, Rodrigues T, Albuquerque I, La prevalencia de la ansiedad dental y su relación con factores socioeconómicos entre las mujeres embarazadas en Joao Pessoa, Brasil, Revista Cubana de Estomatología, Rev Cubana Estomatol vol.51 no.2 Ciudad de La Habana abr.-jun. 2014, Disponible en: http://scielo.sld.cu/scielo.php?pi$\mathrm{d}=\$ 003475072014000200003 \&$ script=sci_arttext\&tIng=pt

21. Álvarez M, Casanova Y, Toledo A, Espeso N, Miedo al tratamiento estomatológico en pacientes de la clínica estomatológica docente provincial, Humanidades Médicas, Rev Hum Med v.7 n.1 Ciudad de Camaguey ene.-abr. 2007, Disponible en: http://scielo.sld.cu/ scielo.php?pid=S172781202007000100006\&script=sci_arttext\&tlng=pt

22. Sanjuán $M$, Intervenciones musicales para la ansiedad odontológica en pacientes pediátricos y adultos, Ene. vol.9 no.2 Santa Cruz de La Palma 2015, Disponible en: http:// scielo.isciii.es/scielo.php?script=sci_arttext\&pid=S1988-348X2015000200011

23. Díaz A, Eugenia M, Raposo S, Pulido R, Técnicas de distracción para reducir la ansiedad en el dentista, Revista europea de odontología, Publicado el: 27/11/2017, Disponible en: http://www.redoe.com/ver.php?id=273

24. Tobón $\mathrm{D}$, Beatriz L, Hoyos B, Comportamiento del nivel de ansiedad y del grado de dolor en pacientes con tratamiento endodóntico, Revista Ces Odontologia, Vol 18, No 1(2005) Disponible en: http://revistas.ces.edu.co/index.php/odontologia/article/view/407

25. Briceño V, Félix E, Niveles De Ansiedad Y Miedo A La Atención Odontológica En Pacientes Adultos Que Acuden A Consulta En La Clínica Estomatológica De Moche De La Universidad Nacional De Trujillo, 2008, Universidad Nacional de Trujillo Fecha: 2009, Disponible en: http://dspace.unitru.edu.pe/handle/UNITRU/401

26. González Ramírez MT, Landero Hernández R, García-Campayo J. Relación entrela depresión, la ansiedad y los síntomas psicosomáticos en una muestra de estudiantes universitarios del norte de México. Rev Panam Salud Publica. 2009;25(2):141-5. Disponible en: http://www.scielosp.org/pdf/rpsp/v25n2/v25n2a07.pdf

27. Ríos Erazo M, Herrera Ronda A, Rojas Alcayaga G. Ansiedad dental: evaluación y tratamiento. Av Odontoestomatol [Internet]. 2014;30(1):39-46. Disponible en: http://scielo. isciii.es/scielo.php?script=sci_arttext\&pid=S0213-12852014000100005

28. Al-Namankany, A.i de Souza, M. \& Ashley, P. Evidence-based dentistry: analysis of dental anxiety scales for children. Br. Dent. J., 212(5):219-22, 2012. Disponible en: https://www. nature.com/articles/sj.bdj.2012.174

29. Cázares F, Montoya B, Quiroga M. "Ansiedad dental en pacientes adultos en el tratamiento odontológico". Revista Mexicana de Estomatología. 2015 Enero; 2(2).

30. Villavicencio Caparó E, Alvear Córdova MC, Cuenca León K, y cols. El tamaño muestral para la tesis ¿Cuántas personas debo encuestar?. Revista OACTIVA UC Cuenca. Vol. 2. No. 1, pp 59-62. 2017. Disponible en: https://www.researchgate.net/publication/323649401

31. Hernández Sampieri R. Metodología de la investigación, Edit. 5 Mc Graw- Hill México 2010. Disponible en: https://www.esup.edu.pe/descargas/dep_investigacion/Metodologia\%20de\%20la\%20investigaci\%C3\%B3n\%205ta\%20Edici\%C3\%B3n.pdf 
32. Rivera I, Fernández A. Ansiedad y miedos dentales en escolares hondureños. Revista Latinoamericana de Psicología. 2005; 37(3).

33. Dhó M. Actitudes de salud bucodental en relación al nivel socoeconómico en individuos adultos. Avances en odontoestomatología. 2015 0ctubre; 31(2).

34. Gracia A, Santabárbara J, López R, Aznar T, Marcos G. Ocupación laboral y riesgo de deterioro cognitivo y demencia en personas mayores de 55 años: una revisión sistemática. Revista Española de Salud Pública. 2016 Junio; 90(21).

35. Lima M, Casanova Y. Miedo, ansiedad y fobia al tratamiento estomatológico. Humanidades Médicas. 2006; 6(1).

36. Rodrigues $\mathrm{H}$. Prevalencia y aspectos sociodemográficos de la ansiedad al tratamiento estomatológico. Revista Cubana de Estomatología. 2016 Octubre; 53(4).

37. Muza R, Muza P. Ansiedad en un servicio de emergencia dental. Horizonte de Enfermería. 2008 Enero; 1(1).

38. Villavicencío-Caparó E, Cuenca KdlÁ. ODNTOLOGÍA ACTIVA.. [Online].; 2016 [cited 2018 Enero 25. Available from:

39. http://oactiva.ucacue.edu.ec/index.php?journal=OACTIVAUCACUE\&page=article\&op=view\&path $\% 5 \mathrm{~B} \% 5 \mathrm{D}=20$

\section{Recibido:}

\section{Aceptado:}

